\title{
Introduction to Spatio-temporal data management and analytics for Smart City research
}

\author{
Shuo Shang ${ }^{1} \cdot$ Lisi Chen ${ }^{2} \cdot$ Christian S. Jensen $^{3} \cdot$ Panos Kalnis ${ }^{4}$
}

Published online: 21 January 2020

(C) Springer Science+Business Media, LLC, part of Springer Nature 2020

This special issue of the GeoInformatica journal covers recent advances in spatio-temporal data management and analytics in the context of smart city and urban computing. It contains 11 articles that present solid research studies and innovative ideas in the area of spatiotemporal data management for smart city research. All of the 11 papers went through several rounds of rigorous reviews by the guest editors and invited reviewers.

Geo-textual query processing has been receiving much attention in area of spatiotemporal data management. The paper, by Xinyu Chen et al., "S2R-tree: a pivot-based indexing structure for semantic-aware spatial keyword search," proposes a pivot-based hierarchical indexing structure to integrate spatial and semantic information in a seamless way. The proposed index is able to return accurate query results that take semantic meaning of geo-textual objects into consideration. Another paper, by Zhongpu Chen et al., "ITISS: an efficient framework for querying big temporal data," proposes an inmemory based two-level index structure in Spark, which is easily understood and implemented, but without loss of effectiveness and efficiency. Additionally, the paper, by Xiaozhao Song et al., "Collective spatial keyword search on activity trajectories," presents an effective and efficient collective spatial keyword query processing algorithm

Shuo Shang

jedi.shang@gmail.com

Lisi Chen

lisi.chen@inceptioniai.org

Christian S. Jensen

csj@cs.aau.dk

Panos Kalnis

panos.kalnis@kaust.edu.sa

1 UESTC, Chengdu, China

2 Inception Institute of Artificial Intelligence, Abu Dhabi, United Arab Emirates

3 Aalborg University, Aalborg, Denmark

4 KAUST, Thuwal, Saudi Arabia 
on activity trajectories. Finally, Lisi Chen et al., "Spatial keyword search: a survey," present a survey of existing studies regarding spatial keyword search.

Location-based social networks (LBSNs) are becoming increasingly indispensable in smart cities. Hao Wang and Ziyu Lu develop the first unified and generic framework to support userpreference based sequence matching in their paper "Preference-aware sequence matching for location-based services." Yanhui Li et al. propose an approach to extracting similar user pattern from LBSNs and annotating semantic tags of locations in their paper "Annotating semantic tags of locations in location-based social networks." The problem is solved by training a binary ELM classifier for each tag in the tag space to support multi-label classification.

Spatial crowdsourcing (SC) is an emerging research direction in spatio-temporal data analytics. Tianshu Song et al. focus on solving a fundamental issue in SC, assigning tasks to suitable workers to obtain multiple global objectives, in their paper "Multi-skill aware task assignment in real-time spatial crowdsourcing." They define the multi-skill aware task assignment problem in real-time SC, which is proven to be NP-hard, and propose an online greedy algorithm that iteratively assigns optimal workers. Yiming Li et al., in their paper "Two-sided online bipartite matching in spatial data: experiments and analysis," present a comprehensive evaluation and analysis of the representative algorithms for the two-sided online bipartite matching problem, which is widely studied in the area of spatio-temporal data management.

Furthermore, the paper, by Yuliang Ma et al., "Graph simulation on large scale temporal graphs," investigates the problem of temporal bounded simulation on temporal graphs, which is a fundamental problem in urban computing. It presents a simulation matching framework consisting of pattern segmentation, temporal bounded simulation of pattern segments, and result integration. Mengqing Mei et al. focus on another fundamental problem in urban computing, identifying the correlation between features and labels from multi-label urban datasets, in their paper "An innovative multi-label learning based algorithm for city data computing." In particular, they propose a multi-label learning algorithm that learns separate subspaces for features and labels by maximizing the independence between the components in each subspace.

Finally, the paper, by Jihai Yang et al., "Joint hyperspectral unmixing for urban computing," focuses on an important problem related to urban computing: joint hyperspectral unmixing. Specifically, it presents an algorithm to process two hyperspectral images, simultaneously, and makes full use of the available information when most of the signals at the two end points are similar.

These papers represent a variety of directions in the fast-growing area of spatio-temporal data management and analytics in smart city applications. We hope that these papers will foster the development of smart cities and inspire more research in this promising area. 